\title{
NOTE ON A THEOREM ON QUADRATIC RESIDUES
}

\section{KAI-LAI CHUNG}

In this note we shall give a short proof of a known result:

Theorem. For every prime $p \equiv 3(\bmod 4)$ there are more quadratic residues $\bmod p$ between 0 and $p / 2$ than there are between $p / 2$ and $p$.

An equivalent statement of this theorem is as follows (see E. Landau, Vorlesungen über Zahlentheorie, vol. 1, p. 129):

Für $p \equiv 3(\bmod 4)$ haben mehr unter den Zahlen $1^{2}, 2^{2}, \cdots,(p-1)^{2} / 4$ ihren Divisionsrest $\bmod p$ unter $p / 2$ als über $p / 2$.

For proof we shall use Fourier series with one of its applications, namely Gaussian sums.

Write $s^{2}=q p+r, 0 \leqq r<p$, so that

$$
\left[\frac{s^{2}}{p}\right]=q
$$

It is evident that we have

$$
\left[\frac{2 s^{2}}{p}\right]-2\left[\frac{s^{2}}{p}\right]=\left\{\begin{array}{lll}
0 & \text { if } & r<p / 2 ; \\
1 & \text { if } & r>p / 2 .
\end{array}\right.
$$

Therefore we have to prove that $\sum_{s=1}^{(p-1) / 2}\left(\left[2 s^{2} / p\right]-2\left[s^{2} / p\right]\right)<(p-1) / 4$, or $\leqq(p-1) / 4$ since $p \equiv 3(\bmod 4)$.

By a well known expansion in Fourier series, we have

so that

$$
x-[x]-\frac{1}{2}=-\sum_{n=1}^{\infty} \frac{\sin 2 n \pi x}{n \pi},
$$

$$
\lfloor x\rfloor=x-\frac{1}{2}+\sum_{n=1}^{\infty} \frac{\sin 2 n \pi x}{n \pi} .
$$

Substituting, we get

$$
\begin{aligned}
{\left[\frac{2 s^{2}}{p}\right]-2\left[\frac{s^{2}}{p}\right]=} & \frac{2 s^{2}}{p}-\frac{1}{2}+\sum_{n=1}^{\infty} \frac{\sin \left(4 n \pi s^{2} / p\right)}{n \pi} \\
& -2\left\{\frac{s^{2}}{p}-\frac{1}{2}+\sum_{n=1}^{\infty} \frac{\sin \left(2 n \pi s^{2} / p\right)}{n \pi}\right\} \\
= & \frac{1}{2}+\sum_{n=1}^{\infty} \frac{1}{n \pi}\left\{\sin \frac{4 n \pi s^{2}}{p}-2 \sin \frac{2 n \pi s^{2}}{p}\right\} ;
\end{aligned}
$$




$$
\begin{aligned}
& \sum_{s=1}^{(p-1) / 2}\left(\left[\frac{2 s^{2}}{p}\right]-2\left[\frac{s^{2}}{p}\right]\right) \\
& \quad=\frac{p-1}{4}+\sum_{n=1}^{\infty} \frac{1}{n \pi}\left\{\sum_{s=1}^{(p-1) / 2}\left(\sin \frac{4 n \pi s^{2}}{p}-2 \sin \frac{2 n \pi s^{2}}{p}\right)\right\} .
\end{aligned}
$$

Therefore we have to prove that

$$
\sum_{n=1}^{\infty} \frac{1}{n \pi} \sum_{s=1}^{(p-1) / 2} \sin \frac{4 n \pi s^{2}}{p} \leqq \sum_{n=1}^{\infty} \frac{2}{n \pi} \sum_{s=1}^{(p-1) / 2} \sin \frac{2 n \pi s^{2}}{p} .
$$

Now we have by the results on Gaussian sums,

$$
\begin{aligned}
& \text { if }\left(\frac{2 n}{p}\right)=1, \quad \sum_{s=1}^{(p-1) / 2} e^{2 n\left(2 \pi i s^{2} / p\right)}=\sum_{(r / p)=1} e^{2 \pi i r / p}=+\frac{i p^{1 / 2}}{2} ; \\
& \text { if }\left(\frac{2 n}{p}\right)=-1, \quad \sum_{s=1}^{(p-1) / 2} e^{2 n\left(2 \pi i s^{2} / p\right)}=\sum_{(r / p)=1} e^{-2 \pi i r / p}=-\frac{i p^{1 / 2}}{2} ; \\
& \text { if }\left(\frac{2 n}{p}\right)=0, \quad \sum_{s=1}^{(p-1) / 2} e^{2 n\left(2 \pi i s^{2} / p\right)}=\frac{p-1}{2} .
\end{aligned}
$$

Taking imaginary parts, we obtain

$$
\sum_{s=1}^{(p-1) / 2} \sin \frac{4 n \pi s^{2}}{p}=\left(\frac{2 n}{p}\right) \frac{p^{1 / 2}}{2} .
$$

Similarly,

$$
\sum_{s=1}^{(p-1) / 2} \sin \frac{2 n \pi s^{2}}{p}=\left(\frac{n}{p}\right) \frac{p^{1 / 2}}{2} .
$$

Therefore we have to prove that

$$
\frac{1}{\pi} \sum_{n=1}^{\infty} \frac{1}{n}\left(\frac{2 n}{p}\right) \frac{p^{1 / 2}}{2} \leqq \frac{2}{\pi} \sum_{n=1}^{\infty} \frac{1}{n}\left(\frac{n}{p}\right) \frac{p^{1 / 2}}{2} ;
$$

that is,

$$
\sum_{n=1}^{\infty} \frac{1}{n}\left(\frac{2 n}{p}\right) \leqq 2 \sum_{n=1}^{\infty} \frac{1}{n}\left(\frac{n}{p}\right)
$$

This is equivalent to the relation

$$
\sum_{n=1}^{\infty} \frac{-1}{n}\left(\frac{n}{p}\right) \leqq 2 \sum_{n=1}^{\infty} \frac{1}{n}\left(\frac{n}{p}\right), \quad \text { if } p \equiv 3(\bmod 8) ;
$$




$$
\sum_{n=1}^{\infty} \frac{1}{n}\left(\frac{n}{p}\right) \leqq 2 \sum_{n=1}^{\infty} \frac{1}{n}\left(\frac{n}{p}\right), \quad \text { if } p \equiv 7(\bmod 8) .
$$

Thus in each case we have to prove that

$$
\sum_{n=1}^{\infty} \frac{1}{n}\left(\frac{n}{p}\right) \geqq 0 \text {. }
$$

Now Landau would call this last result trivial. But for the sake of completeness we give its proof here; we have in fact, for $s>1$,

$$
\left\{\sum_{n=1}^{\infty} \frac{1}{n^{s}}\left(\frac{n}{p}\right)\right\} \prod_{p_{1}}\left(1-\frac{1}{p_{1}^{s}}\left(\frac{p_{1}}{p}\right)\right)=1,
$$

where $p_{1}$ runs through the sequence of primes. The series being uniformly convergent for $s \geqq 1$ its sum is continuous at $s=1$. Hence the result follows.

Tsing-Hua University, Kunming, China 